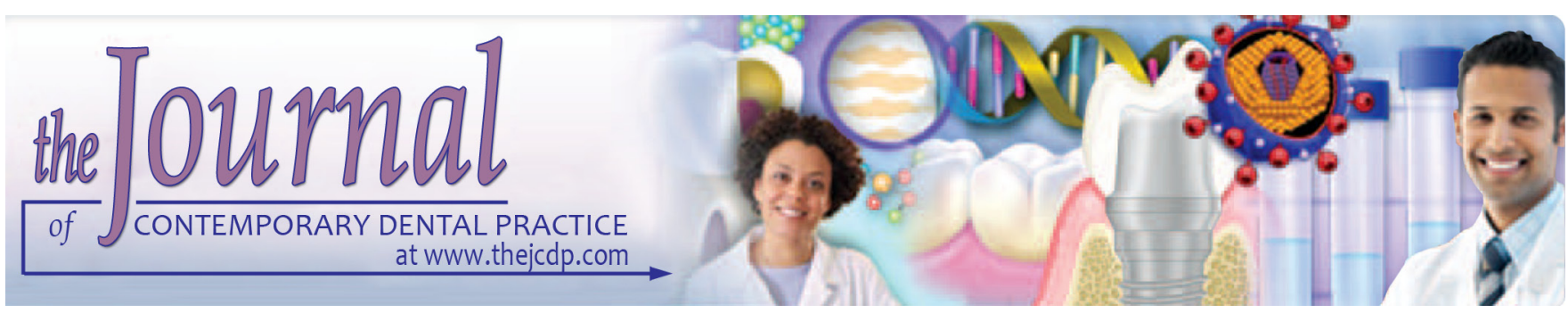

\title{
Correlation of Dental and Skeletal Malocclusions in Sagittal Plane among Saudi Orthodontic Patients
}

\author{
${ }^{1}$ Nasir Al-Hamlan, ${ }^{2}$ Balsam Al-Eissa, ${ }^{3}$ Ahmad S Al-Hiyasat, ${ }^{4}$ Farraj S Albalawi, ${ }^{5}$ Anwar E Ahmed
}

\begin{abstract}
Aim: Whether or not the dental relationship correlates with skeletal relationship in the sagittal plane is an area of interest for orthodontic diagnosis and treatment planning. Thus, the aim of this study was to investigate the correlation of the dental malocclusion and the skeletal malocclusion in the sagittal plane among Saudi orthodontic patients.
\end{abstract}

Materials and methods: Orthodontic dental casts and cephalometric radiographs of 124 patients were investigated and analyzed. The dental casts were classified in relation to the molar relationship according to Angle's classification and to the incisal relationship according to the British Standards Institution (BSI) classification. The sagittal relation in the cephalometric radiographs was analyzed according to ANB angle and WITS appraisal.

Results: The results show that the incisal relation had a very high significant association with WITS appraisal ( $p=0.0045)$, whereas with ANB, the association was marginally significant $(p=0.0528)$. No significant associations were found with molar relation neither at ANB $(p=0.2075)$ nor at the WITS $(p=0.4794)$ appraisal. Significant positive correlations between ANB and WITS appraisal were found at the three incisal classification classes (class I, $r=0.73$; class II, $r=0.64$; class III, $r=0.75$ ) and no significant correlation was observed in all classes with the Angle's (molar) classification.

Conclusions: The incisal classification had a significant association with WITS appraisal, whereas with ANB the association was marginally significant.

\footnotetext{
${ }^{1-4}$ Department of Dental Services, King Abdulaziz Medical City and College of Dentistry, King Saud Bin Abdulaziz University for Health Sciences; National Guard Health Affairs, Riyadh Saudi Arabia

${ }^{5}$ Department of Epidemiology and Biostatistics, College of Public Health and Health Informatics; King Saud Bin Abdulaziz University for Health Sciences; National Guard Health Affairs Riyadh, Saudi Arabia
}

Corresponding Author: Ahmad S Al-Hiyasat, Professor Faculty of Dentistry, Jordan University of Science and Technology, PO Box 3030, Irbid 22110, Jordan, Phone: +962795-843677, e-mail: hiyasat@just.edu.jo
No correlation was found between Angle's (molar) classification and ANB or WITS appraisal.

Clinical significance: The incisal relation could be considered as a good indicator of the skeletal malocclusion in the sagittal plane in the orthodontic practice.

Keywords: Dental malocclusions, Skeletal malocclusions, Sagittal plane.

How to cite this article: Al-Hamlan N, Al-Eissa B, Al-Hiyasat AS, Albalawi FS, Ahmed AE. Correlation of Dental and Skeletal Malocclusions in Sagittal Plane among Saudi Orthodontic Patients. J Contemp Dent Pract 2015;16(5):353-359.

\section{Source of support: Nil}

Conflict of interest: None

\section{INTRODUCTION}

Diagnosis of malocclusion is an important criterion for the success of any orthodontic treatment and, therefore, it is essential for the orthodontist to have adequate knowledge of dental occlusion and the underlying skeletal relationship of the patient to reach the proper diagnosis and treatment plan of the malocclusion.

A good method of recording or measuring malocclusion is essential for documentation of the prevalence and severity of malocclusion in different populations and also will help in education and classifying the various types of malocclusion. ${ }^{1}$ The earliest published method of recording and classifying malocclusion was Angle's classification in $1899,{ }^{2}$ which was based on the anteroposterior relationship of the maxillary and mandibular first molars and the alignment of all the teeth to the line of occlusion. ${ }^{1,2}$ Angle's classification has become the most widely used classification in orthodontics for diagnosis and treatment planning; however, it has a drawback that it is representing only the dental relationship in the sagittal plane and not the skeletal relationship. ${ }^{3}$ 
Indeed, previous studies revealed that the dental arch relationship is largely affected by the facial skeleton configuration upon which the teeth are invested. ${ }^{4,5}$ Thus, studies have investigated the relationship between the anteroposterior dental arch and jaw-base relationships. ${ }^{6-8}$ Both angular and linear measurements have been proposed in the assessment of anteroposterior jaw-base relationship. ${ }^{6,9-12}$ Whether the anteroposterior dental arch correlates with the jaw-base relationship and also whether both need to be assessed in orthodontic diagnosis and treatment planning is an area of debate. Thus, the relationships between craniofacial morphology and malocclusion have long been of interest to orthodontists. ${ }^{13,14}$ Another parameter that is of importance in occlusal analysis is the incisal relationship and the overjet, which is one of the parameters used to assess the sagittal relationship of the upper and lower dental arch. The cause of large or small overjet could also be skeletal, dental or a combination of both. ${ }^{15}$ Therefore, the aim of this study was to investigate the correlation of the skeletal malocclusion with dental malocclusion in the sagittal plane, which includes molar relation and incisal relation, in Saudi orthodontic patients. The hypothesis is the incisor relation has a strong correlation with the skeletal pattern than molar relation.

\section{MATERIALS AND METHODS}

A random sample of 350 records of patients who had attended the Dental Center for Orthodontic treatment were selected from the Patients' Record Department at King Abdulaziz Medical City, National Guard Health Affair, Riyadh, Kingdom of Saudi Arabia. The lateral cephalometric radiographs and study casts from the patients' records were reviewed and only 124 cases were found to fulfill the inclusion criteria which require the following:

- Proper study model that has no breakage or chipped

- All permanent maxillary and mandibular teeth are present from first molar to first molars

- No previous orthodontic treatment

- No missing or impacted teeth or retained primary teeth

- No broken tooth or large restoration (which replace one cusp or more or involvement of the incisal third or a crown)

- Cases with rotated two central incisors more than 45 degree in one jaw or both were excluded

- All lateral cephalometric radiographs must be in rest position.

The patients' records were treated with privacy and the examiners were trained and with experience in orthodontics and occlusion analysis. The research protocol was registered and approved by King Abdullah Research Center in King Abdulaziz Medical City.

Three examiners carried out the analysis of the cephalomertic radiographs and the study models of the cases that were chosen and approved, independently as follows:

The first examiner performed the cephalometric tracing and the measurement of the ANB and WITS analysis. ${ }^{10,11}$

The second examiner assessed the molar occlusion relationship according to Angle's classification, ${ }^{16}$ as class I, class II, and class III, half-unit class II or class III were recorded as class II or class III, respectively. Molar area was then covered bucally by a tape so the third examiner was not able to see the molar relation.

The third examiner assessed the incisor occlusion relationship with the tape covering the molar area. Incisal relation was assessed according to the British Standards Institution (BSI) definition ${ }^{17}$ which has been used previously for occlusion classification as follows: ${ }^{18}$

Class I: The lower incisor edges occlude with or lie immediately below the cingulum plateau of the upper central incisors.

Class II (div. 1): The lower incisor edges lie posterior to the cingulum plateau of the upper incisors. Upper incisors are normal inclined or proclined and the overjet is increased.

Class II (div. 2): The lower incisor edges lie posterior to the cingulum plateau of the upper incisors. Upper incisors are retroclined and overjet is usually minimal but may be increased.

Class III: The lower incisor edges lie anterior to the cingulum plateau of the upper incisors. The overjet is reduced or reversed.

\section{STATISTICAL ANALYSIS}

Data were collected and filled in special coded forms that were prepared for this purpose; one for the cephalometric analysis and measurement, and the second for the study cast analysis (molar and incisal relationship).

The data were then transferred from the coded sheets to a personal computer and analyzed using SAS program (versions 9.2, SAS Institute Inc, Cary, NC, USA). Frequencies and percentages were used to summarize categorical variables.

Chi-square tests were used to test the association between occlusal characteristics and the cephalometric measurements. If the large sample assumption was violated, Fisher's exact test would be employed. Pearson correlation coefficients were used to examine the relationship between the sagittal skeletal parameters 
measured from the lateral cephalometric radiograph (ANB and WITS) and the dental relationship of the molar occlusion and the incisor occlusion measured from the cast model. $\mathrm{p}$ values considered to be statistically significant at the 0.05 level or less.

\section{RELIABILITY}

Intraexaminer reliability in assessing the orthodontic models was tested by having the same examiner reexamine 14 randomly chosen orthodontic models at an interval of 1 month. Test-retest reliability kappa ( $\kappa)$ statistics revealed that there was a substantial agreement between test and retest with respect to incisor classification, kappa was $0.75(\mathrm{p}<0.001)$. There was almost perfect agreement between test and retest in molar right and left classification, kappa was 0.81 and 0.80 respectively $(\mathrm{p}<0.001)$.

\section{RESULTS}

From the 124 cases that were investigated in the present study, there were 53 cases with incisal class II of which only two cases with class II division 2 and 51 cases with class II division 1; therefore, all the class II cases were analyzed statistically together as class II incisor relationship with class I and class III cases.

There were 24 cases where the right side was not matching the left side in molar classification, these cases were classified as subdivision [16 cases $(12.90 \%)$ had class I with class II, 3 cases $(2.42 \%)$ had class I with class III, and 5 cases $(4.03 \%)$ had class II with class III].

The age of the subjects involved in this study; ranged from 12 to 40 years old with the mean age of $18 \pm 5$.

Table 1 presented the demographics and clinical characteristics of the dental (incisal and molar relation) and skeletal (ANB and WITS) malocclusion classification. The Table 1 shows that $59 \%$ of the cases were females and $41 \%$ were males.

Relating the incisor classification vs molar classification, there was 28 vs 50\% for class I, 43 vs 16\% for class II, and 29 vs 11\% for class III, respectively. This indicates that most of the cases in molar classification were class I (50\%), whereas cases with incisal classification were class II $(43 \%)$. Also, $19 \%$ of the cases in molar classification had one side class I and the other side either class II (12.90\%) or class III (2.42\%) molar relation, a combination of class II with class III was also found in $(4.03 \%)$.

When reviewing the ANB classification vs WITS classification there was 52 vs 31\% for class I, 35 vs 37\% for class II and 13 vs 32\% for class III, respectively. This also indicate that most of the cases in ANB classification were class I (52\%), whereas in WITS classification (37\%)
Table 1: Demographics and clinical characteristics of dental and skeletal malocclusion classification $(\mathrm{N}=124)$

\begin{tabular}{lll}
\hline Variable & Characteristics & Number (\%) \\
\hline Gender & Female & $73(58.9)$ \\
Incisor relation & Male & $51(41.1)$ \\
& Class I & $35(28.2)$ \\
& Class II & $53(42.7)$ \\
Molar relation & Class III & $36(29.0)$ \\
& Class I & $66(53.2)$ \\
& Class II & $20(16.1)$ \\
& Class III & $14(11.3)$ \\
& Subdivision & $24(19.4)$ \\
& Class I with class II & $16(12.90)$ \\
ANB & Class I with class III & $3(2.42)$ \\
& Class II with class III & $5(4.03)$ \\
& Class I & $65(52.4)$ \\
WITS & Class II & $43(34.7)$ \\
& Class III & $16(12.9)$ \\
& Class I & $38(30.7)$ \\
& Class II & $46(37.1)$ \\
& Class III & $40(32.3)$ \\
\hline
\end{tabular}

were class II which was not far much than class III (32\%) or class I (31\%).

Table 2 showed the statistical comparison of the demographic and clinical characteristics across the ANB classification level. It revealed that there was a highly significant association with WITS classification $(\mathrm{p}=$ $0.0001)$, but not with molar classification $(\mathrm{p}=0.2075)$, whereas with incisor classification there was a $94.72 \%$ chance that the association to be true $(p=0.0528)$ which is marginally significant. When the comparison was carried out at the Wits classification level (Table 3), a highly significant association was found between WITS classification and incisor classification ( $p=0.0045)$.

The analysis of the data was carried out to determine the correlation between molar relation, ANB, and Wits for each incisor class (class I, class II and class III). The results were presented in Tables 4, 5 and 6 ( $a$ and b) respectively.

Statistically significant positive correlations between ANB and WITS were observed in incisor class I $(r=0.73)$, incisor class II $(r=0.64)$, and incisor class III $(r=0.75)$. That's as ANB increases, the WITS also increases and vice versa.

Nonsignificant negative correlations between molar relation and ANB or WITS were observed in incisor class I $(\mathrm{r}=-0.25, \mathrm{r}=-0.11$, respectively), and incisor class III $(\mathrm{r}=-0.10, \mathrm{r}=-0.01$, respectively). On the contrary, a nonsignificant positive correlation was found between molar relation and ANB or WITS at Incisor class II $(r=0.03, r=0.06$, respectively).

Overall, a significant positive correlation was found between the ANB and WITS but not with molar relation at any of the three incisor classes. 
Table 2: Comparison of demographic and clinical characteristics across ANB levels

\begin{tabular}{|c|c|c|c|c|c|c|}
\hline \multirow[b]{2}{*}{ Variable } & \multirow[b]{2}{*}{ Characteristics } & \multicolumn{3}{|c|}{$A N B$} & \multirow{2}{*}{$\begin{array}{l}\text { Chi-square/Fisher } \\
\text { tests }\end{array}$} & \multirow[b]{2}{*}{$p$-value } \\
\hline & & Class I & Class II & Class III & & \\
\hline \multirow[t]{2}{*}{ Gender } & Female & 36 (49.32) & 29 (39.73) & $8(10.96)$ & 2.1509 & 0.3411 \\
\hline & Male & $29(56.86)$ & $14(27.45)$ & $8(15.69)$ & & \\
\hline \multirow[t]{3}{*}{ Incisor relation } & Class I & $18(51.43)$ & $12(34.29)$ & $5(14.29)$ & 8.9195 & $0.0528^{*}$ \\
\hline & Class II & $23(43.40)$ & $25(47.17)$ & $5(9.43)$ & & \\
\hline & Class III & $24(66.67)$ & $6(16.67)$ & $6(16.67)$ & & \\
\hline \multirow[t]{4}{*}{ Molar relation } & Class I & $33(50.00)$ & $25(37.88)$ & $8(12.12)$ & 7.9680 & 0.2075 \\
\hline & Class II & $12(60.00)$ & $8(40.00)$ & $0(0.00)$ & & \\
\hline & Class III & $8(57.14)$ & $2(14.29)$ & $4(28.57)$ & & \\
\hline & Subdivision & $12(50.00)$ & $8(33.33)$ & $4(16.67)$ & & \\
\hline \multirow[t]{3}{*}{ Wits } & Class I & 25 (65.79) & $11(28.95)$ & $2(5.26)$ & 54.8053 & $0.0001^{\text {** }}$ \\
\hline & Class II & $15(32.61)$ & $31(67.39)$ & $0(0.00)$ & & \\
\hline & Class III & $25(62.50)$ & $1(2.50)$ & $14(35.00)$ & & \\
\hline
\end{tabular}

* There is a $94.72 \%$ chance that the association between level of incisor and ANB classes is true.

**The Chi-square/Fisher's exact test is significant at the 0.05 level.

Table 3: Comparison of demographic and clinical characteristics across Wits levels

\begin{tabular}{|c|c|c|c|c|c|c|}
\hline \multirow[b]{2}{*}{ Variable } & \multirow[b]{2}{*}{ Characteristic } & \multicolumn{3}{|c|}{ WITS } & \multirow[b]{2}{*}{ Chi-square/ Fisher tests } & \multirow[b]{2}{*}{$p$-value } \\
\hline & & Class I & Class II & Class III & & \\
\hline \multirow[t]{2}{*}{ Gender } & Female & $22(30.14)$ & $26(35.62)$ & $25(34.25)$ & 0.3374 & 0.8448 \\
\hline & Male & $16(31.37)$ & $20(39.22)$ & $15(29.41)$ & & \\
\hline \multirow[t]{3}{*}{ Incisor relation } & Class I & $12(34.29)$ & $14(40.00)$ & $9(25.71)$ & 15.1212 & $0.0045^{*}$ \\
\hline & Class II & $16(30.19)$ & $26(49.06)$ & $11(20.75)$ & & \\
\hline & Class III & $10(27.78)$ & $6(16.67)$ & $20(55.56)$ & & \\
\hline \multirow[t]{4}{*}{ Molar relation } & Class I & $19(28.79)$ & $25(37.88)$ & $22(33.33)$ & 5.5168 & 0.4794 \\
\hline & Class II & $6(30.00)$ & $10(50.00)$ & $4(20.00)$ & & \\
\hline & Class III & $5(35.71)$ & $2(14.29)$ & $7(50.00)$ & & \\
\hline & Subdivision & $8(33.33)$ & $9(37.50)$ & $7(29.17)$ & & \\
\hline
\end{tabular}

${ }^{*}$ The Chi-Square/Fisher's exact test is significant at the 0.05 level. There is an association between level of incisor and WITS classes

Table 4: (a) Demographics and clinical characteristics for incisor class I, (b) incisor class I correlation coefficients

(a) Demographics and clinical characteristics for incisor class I $(\mathrm{N}=35)$

\begin{tabular}{lll}
\hline Variable & Characteristics & Number (\%) \\
\hline Gender & Female & $20(57.14)$ \\
Molar relation & Male & $15(42.86)$ \\
& Class I & $23(65.71)$ \\
& Class II & $4(11.43)$ \\
& Class III & $2(5.71)$ \\
ANB & Subdivision & $6(17.14)$ \\
& Class I & $18(51.43)$ \\
WITS & Class II & $12(34.29)$ \\
& Class III & $5(14.29)$ \\
& Class I & $12(34.29)$ \\
& Class II & $14(40.00)$ \\
(b) Incisor class I correlation coefficients (N = 35) \\
\hline Variable & Class III & $9(25.71)$ \\
\hline Molar Relation & -0.25 & WITS \\
WITS & $0.73^{*}$ & -0.11 \\
\hline$*$
\end{tabular}

${ }^{*}$ Pearson correlation is significant at the 0.05 level.
Table 5: (a) Demographics and clinical characteristics for incisor class II, (b) incisor class II correlation coefficients

(a) Demographics and clinical characteristics for incisor class II $(\mathrm{N}=53)$

\begin{tabular}{lll}
\hline Variable & Characteristics & Number $(\%)$ \\
\hline Gender & Female & $34(64.15)$ \\
\multirow{3}{*}{ Molar relation } & Male & $19(35.85)$ \\
& Class I & $28(52.83)$ \\
& Class II & $13(24.53)$ \\
& Class III & $2(3.77)$ \\
ANB & Subdivision & $10(18.87)$ \\
& Class I & $23(43.40)$ \\
\multirow{2}{*}{ WITS } & Class II & $25(47.17)$ \\
& Class III & $5(9.43)$ \\
& Class I & $16(30.19)$ \\
& Class II & $26(49.06)$ \\
& Class III & $11(20.75)$
\end{tabular}

(b) Incisor class II correlation coefficients $(N=53)$

\begin{tabular}{lll}
\hline Variable & ANB & WITS \\
\hline Molar Relation & 0.03 & 0.06 \\
WITS & $0.64^{*}$ & \\
\hline
\end{tabular}

* Pearson correlation is significant at the 0.05 level. 
Table 6: (a) Demographics and clinical characteristics for incisor class III, (b) incisor class III correlation coefficients

\begin{tabular}{lll}
\hline \multicolumn{3}{c}{$\begin{array}{c}\text { (a) Demographics and clinical characteristics } \\
\text { for incisor class III }(\mathrm{N}=36)\end{array}$} \\
\hline Variable & \multicolumn{1}{c}{ Characteristics } & Number (\%) \\
\hline Gender & Female & $19(52.78)$ \\
Molar relation & Male & $17(47.22)$ \\
& Class I & $15(41.67)$ \\
& Class II & $3(8.33)$ \\
ANB & Class III & $10(27.78)$ \\
& Subdivision & $8(22.22)$ \\
& Class I & $24(66.67)$ \\
WITS & Class II & $6(16.67)$ \\
& Class III & $6(16.67)$ \\
& Class I & $10(27.78)$ \\
& Class II & $6(16.67)$ \\
& Class III & $20(55.56)$
\end{tabular}

(b) Incisor class III correlation coefficients $(\mathrm{N}=36)$

\begin{tabular}{lll}
\hline Variable & ANB & WITS \\
\hline Molar relation & -0.10 & -0.01 \\
WITS & $0.75^{*}$ & \\
\hline
\end{tabular}

* Pearson correlation is significant at the 0.05 level

\section{DISCUSSION}

Previously, it has been reported that skeletal relationship in the sagittal plane do not always correspond with dental relationships, and most of the disagreement has been found in class I dental relationships. ${ }^{7,15}$ Thus, it was proposed that a population-based study is required to strengthen the evidence as whether or not the anteroposterior dental arch relationship is correlated with the jaw-base relationship. ${ }^{14}$ In the previous population study, the correlation between anteroposterior dental arch relationship that was based in the molar relation only was correlated to the anteroposterior jaw-base relationship (skeletal) that was assessed by angular (ANB) and linear (WITS) measurements; ${ }^{14}$ whereas, other study correlate the overjet only with skeletal relationship based also in the angular ANB and linear Wits measurements. ${ }^{15}$ Therefore, our study was design to assess the correlation between the dental arch malocclusion which includes the molar relationship and incisal relationship with the skeletal malocclusion of the jaws that include the angular assessment ANB and linear assessment WITS among the Saudi orthodontic patients. Interesting to notice in our result that large discrepancy between molar classification and incisal classification, 50\% of the samples that were investigate were classified as class I molar relationship vs only $28 \%$ in the incisal classification as class I incisal, whereas for class II $16 \%$ was found to be with class II molar classification vs $43 \%$ as incisal class II classification, this demonstrate the large disagreement between the molar classification and the incisal classification. Thus, for dental arch relation both molar and incisal has to be considered.

Routinely, ANB angle and WITS appraisal are the most common cephalometric tools for assessing the anteroposterior jaw discrepancies. ${ }^{19}$ ANB is not always a reliable measure of the jaw relationships in the sagittal plane, thus WITS appraisal was (may be) used in order to obtain supplementary information. ${ }^{15}$ Therefore, the combination of both ANB and WITS (each) complementing each other and can diagnose skeletal discrepancies and address treatment strategies. ${ }^{20}$

The result of the present study revealed that there was strong association between ANB and WITS classes $(\mathrm{p}=0.0001)$. Highly significant association was also found between incisor class and WITS class ( $p=0.0045)$, whereas with ANB the association was marginally significant $(p=0528)$. The fact that both ANB and WITS assess the same skeletal discrepancy which was proved or supported by the present study result. This finding was in accordance with previous report. ${ }^{3}$ However, the correlation between both in other previous studies was found not as strong as expected. ${ }^{19-21}$

Lack of consistency between ANB and WITS assessments was found in high occlusal plane angle patients. ${ }^{19}$ Earlier study has suggested that in extreme or controversial cephalometric interpretations of the ANB angle and WITS appraisal, a visual inspection provides an essential aid in diagnosis and skeletal classification. ${ }^{22}$

Furthermore, our results show no significant association between molar classes and ANB angle nor with WITS appraisal. This finding showed the lack of correlation between Angle's (molar) classification of the dental malocclusion and the skeletal malocclusion assessed by ANB angle and Wits apprasial. Thus, the result of the present investigation is in disagreement with the findings of Shrikant et al, ${ }^{3}$ who reported a significant positive correlation of the ANB angle and WITS apprasial when applied to the Angle's three groups of malocclusions class I, II, and III. But, is in agreement with Zhou et al, ${ }^{14}$ who reported the evidence that Angle's classification of occlusion, based on dental arch relationship alone will not reveal the full picture of dentofacial deformity, not even in the sagittal plane. On the contrary, the result of the present study indicates that the association was highly significant between the incisal relation with Wits appraisal $(p=0045)$ and marginally significant with ANB $(\mathrm{p}=0528)$. This is in line with Zhou et $\mathrm{al}^{14}$ report indicating that Wits appraisal which is based on linear measurement of anteroposterior jaw-base relationship 
$(p<0.001)$ is more valid reflection of the dental arch relationship than the ANB angular measurement $(p<0.01)$. Although significant positive correlations between ANB and WITS were found in the present study at all incisor classes, as ANB values increases, the WITS values also increases and vice versa. However, the correlation was not significant at all with molar relation at any of the incisal classes. It was reported that the prediction between the variables of ANB and Wits is moderately independent of the dental arch relationship of the molar Angle's classes. ${ }^{14}$

Further, Zupancic et $\mathrm{al}^{15}$ found that in class II division I incisal relation, overjet is a significantly good predictor of the sagittal skeletal relationship. In the present study result, (our study) 53 cases out of 124 of the cases (samples) were class II incisal classification, of which 2 cases (samples) were class II (with) division 2 and 51 cases were class II (with) division I. But both classes were considered as class II for the statistical analyses. Furthermore, Pancherz et $\mathrm{al}^{23}$ reported that with exception of the maxillary incisor position, no basic difference in dentoskeletal morphology exist between class II division 1 and class II division 2 malocclusion.

There was significant correlation between ANB angle and WITS appraisal, but it appeared that Wits appraisal is more realistic in diagnosis of the skeletal malocclusion in the sagittal plane. This was confirmed with the findings of Ucheonye et al. ${ }^{24}$ They stated that 'The difference in diagnostic accuracy between the ANB angle and the Wits appraisal 'shows the WITS appraisal as being more reliable than the ANB in assessment of sagittal dental base discrepancy and based on this, it is wise to use the Wits appraisal as a moderator of the ANB angle in the management of orthodontic patients'.

The results of the present investigation demonstrate strong correlation between the incisal classes of occlusion and the skeletal relationship in the sagittal plane; thus, the results were in accordance and supporting the study hypothesis.

\section{CONCLUSION}

On the basis of cephalometric and study model analysis, the following could be concluded from this study:

- Highly significant association was found between incisal classification and WITS appraisal classification of the jaws ( $p=0.0045)$. But the association was marginally significant with ANB classification $(\mathrm{p}=0.053)$.

- There was no significant association between molar classification and skeletal morphology in the sagittal plane determined by WITS appraisal and ANB angle.

\section{Clinical Significance}

The incisal relation of the occlusion could be considered as a good indicator for diagnosis of the skeletal malocclusion in the sagittal plane in the orthodontic dental practice.

\section{REFERENCES}

1. Hassan R, Rahimah AK. Occlusion, malocclusion and method of measurements-an overview. Arch Orofac Sci 2007;2:3-9.

2. Angle EH. Classification of malocclusion. Dental Cosmos 1899;4:248-264.

3. Shrikant S, Ganapathy KK, Reddy RPR, Thomas M. Correlation of the anteroposterior relationships of the dental arch and jaw-base in subjects with class I, class II and class III malocclusions. Int J Contemp Dent 2011;2:68-73.

4. Bjork A. The nature of facial prognathism and its relation to normal occlusion of the teeth. Am J Orthod 1951;137:106-124.

5. Enlow DH, Kuroda T, Lewis AB. The morphological and morphogenetic basis for craniofacial form and pattern. Angle Orthod 1971;41:161-188.

6. Kim YH, Vietas JJ. Anteroposterior dysplasia indicator: an adjunct to cephalometric differential diagnosis. Am J Orthod 1978;73:619-633.

7. Milacic M, Markovic M. A comparative occlusal and cephalometric study of dental and skeletal anteroposterior relationship. Br J Orthod 1983;10:53-54.

8. Taylor CM. Changes in the relationship of nasion, point A, and point B and the effect upon ANB. Am J Orthod 1969; 56:143-163.

9. Downs WB. Variations in facial relationship: their significance in treatment and prognosis. Am J Orthod 1948;34:812-840.

10. Steiner CC. Cephalometrics for you and me. Am J Orthod 1953;39:729-755

11. Jacobson A. The 'WITS' appraisal of jaw disharmony. Am J Orthod 1975;67:125-138.

12. Kirchner J, Williams S. A comparison of five different methods for describing sagittal jaw relationship. Br J Orthod 1993;20:13-17.

13. Proffit WE, Fields HW, Sarver DM. Contemporary Orthodontics. 4th ed. St Louis, Mo: Mosby Year Book 2007. p. 194.

14. Zhou L, Mok C-W, Hagg U, McGrath C, Bendeus M, Wu J Anteroposterior dental arch and jaw-base relationships in a population sample. Angle Orthod 2008;78:1023-1029.

15. Zupancic S, Pohar M, Farcni kF, Ovsenik M. Overjet as a predictor of sagittal skeletal relationships. Eur J Orthod 2008; 30:269-273.

16. Angle EH. Treatment of malocclusion of the teeth. 7th ed. Philadelphia (PA): SS White Manufacturing Company; 1907.

17. British Standard Institution. British standard glossary of dental terms, BS, 4492. London: HMSO; 1983.

18. Al-Hiyasat AS, Abu-Alhaija ESJ. The relationship between static and dynamic occlusion in 14-17-year-old school children. J Oral Rehabil 2004;31:628-633.

19. Del Santo M Jr. Influence of occlusal plane inclination on ANB and WITS assessment of anteroposterior jaw relationships. Am J Orthod Dentofacial Orthop 2006;129:641-648.

20. Bishara SE, Fahl JA, Peterson LC. Longitudinal changes in the ANB angle and WITS appraisal: clinical implications. Am J Orthod 1983;84:133-139. 
21. Polk CE. A new index for evaluating horizontal skeletal discrepancies and predicting treatment outcomes. Am J Orthod Dentofac Orthop 2003;124:663-669.

22. Hurmerinta K, Rahkamo A, Haavikko K. Comparison between cephalometric classification methods for sagittal jaw relationships. Eur J Oral Sci 1997;105:221-227.
23. Pancherz H, Zieber K, Hoyer B. Cephalometric characteristics of class II division 1 and class II division 2 malocclusions: a comparative study in children. Angle Orthod 1997;67:111-120.

24. Ucheonye IJ, Tokunbo AA, Donald OO. The WITS appraisal among a Nigerian sub-population: an assessment of dental base geometric factors. Braz J Oral Sci 2013;12:275-279. 